\title{
Cultivating Mindfulness: a Comprehensive Approach
}

\author{
William L. Mikulas
}

Published online: 11 September 2014

(C) Springer Science+Business Media New York 2014

I have been a student, teacher, and researcher of mindfulness for over four decades (e.g., Mikulas 1981, 1990, 2002, 2011). As a university professor, I regularly teach a course in Buddhist psychology where mindfulness is a continuous part of the lab. I also regularly teach related free community programs, which provide a wider range of type of students for me to address and learn from. As a visiting professor and member of international organizations interested in the interfacing of Eastern and Western psychologies, I have given many mindfulness-related talks and workshops around the world. In this paper, "students" include all these different groups, and it includes me as well.

My mindfulness-related research has primarily been done by my thesis students. Most of the studies focused on how mindfulness facilitates self-control, as control of anxiety and anger. In conjunction with colleague Bruce Dunn, we did a few studies looking at biological correlates of mindfulness, primarily brainwave frequency and event-related potentials (e.g., Dunn et al. 1991; Mikulas 2000). Decades ago, over many years, we developed a mindfulness questionnaire, now called an awareness questionnaire, which is used in most of our studies.

The purpose of this paper is to share some of what I have learned about teaching mindfulness to a wide range of people. Included in this discussion is what I consider very important points that have received inadequate attention in the professional and popular literatures. The reason for this paper at this time is because of the recent publication of my book Taming the Drunken Monkey (Mikulas 2014b). This allows me to discuss general issues in this paper, with Taming providing, for those interested, the step-by-step way to do what I am discussing. Taming is written for the proverbial intelligent layman and provides instruction in mindfulness and related practices. A recent meta-analysis and review of mindfulness-

W. L. Mikulas $(\bowtie)$

Department of Psychology, University of West Florida, 11000 University Parkway, Pensacola, FL 32514-5750, USA

e-mail: wmikulas@uwf.edu based self-help interventions concludes, "people may be able to successfully develop mindfulness and acceptance skills through interventions that require little or no therapist resource" (Cavanagh et al. 2014, p. 128). Taming is also intended to be used by therapists, researchers, and instructors.

For simplicity of discussion, I will assume the ideal case where I am not constrained by time or topic. This is true in my Buddhist course and with people using Taming on their own. In other situations, such as therapy or research, one may be limited in time, constrained by what topics are appropriate, and/or required to embed mindfulness in other components. For example, I will advocate doing breathwork before mindfulness, but this may be impractical or confounding in some situations. Similarly, Taming uses our 45-item mindfulness/ awareness questionnaire, but many researchers have reported choosing a measurement of mindfulness that is short because they are giving their subjects a number of tests.

\section{Concentration and Awareness}

Mindfulness and all the world's major meditation traditions are based on two fundamental behaviors of the mind: concentration and awareness. These two behaviors are distinctively different in terms of how they are cultivated, their psychological effects, and their neurological correlates (Mikulas 2002).

Concentration is the learned control of the focus of one's attention; it is the behavior of keeping one's awareness, with varying degrees of one-pointedness, on a particular set of contents of the mind, such as a meditation object. Concentration is developed by continually, gently, and firmly bringing one's attention back to the object. This practice develops control of the focus of attention and eventually leads to more control of the contents of the mind and the ability to disidentify with the contents. In addition, if one sits quietly during concentration, the mind becomes calm and relaxed, which usually relaxes the body.

Awareness, as a behavior of the mind, is the active maximizing of the breadth and clarity of awareness. The behavior 
is cultivated by simply noticing whatever arises in consciousness, while minimizing getting lost in related thoughts, reactions, and elaborations. It is just observing the contents and processes of the mind. It is not thinking, categorizing, judging, accepting, or any other cognitive process. It is directly being aware of these processes. Developing awareness facilitates selfcontrol of body, behaviors, feelings, and mind. In Buddhism, it is suggested that this awareness leads to insight (prajna), the ultimate purpose of most Buddhist practices.

Concentration and awareness interact in complex ways, including that an improvement in one often improves the other. In my experience, it is usually best if students develop some concentration before beginning awareness training. It is harder to be aware if the mind is too active like a drunken monkey.

\section{Drunken Monkey}

The Asian metaphor of the untrained mind being like a drunken monkey is one of the most powerful metaphors/ images I know. In teaching concentration, I always begin with a detailed discussion of the activities of the monkey. Students enjoy and quickly relate to this metaphor (a few students have preferred the image of a dog or horse, which they know better).

Thinking about the mind as a drunken monkey facilitates perceiving the activities of the mind more objectively, one is less likely to identify with the contents or processes of the mind. In Taming, the monkey metaphor is used in many ways. For example, various obstacles and distractions to mental training are described as "monkey tricks," things the monkey does to keep from being tamed. Thus, if the mind says that the practices of mental training are "wasting time," the student is more likely to recognize this as a monkey trick rather than a valid reason to stop practicing.

\section{Mindfulness?}

Currently, there is some discussion about exactly what "mindfulness" is. My position is that in Buddhism, it ultimately is awareness. It is certainly different than concentration (cf. eight-fold path, seven factors of enlightenment, the path of insight vs. the path of concentration/absorption). However, the Buddha's descriptions of how to cultivate mindfulness are a practical blend of awareness and concentration, thus allowing for some combining of two very different processes.

Presently, the biggest problem in Western academic and popular approaches to mindfulness is the confusing and confounding of awareness and concentration (cf. Mikulas 2011). The majority of Western research, programs, and assessment tests are severely limited by this confusion. Thus, however, one chooses to define mindfulness; it is extremely important that training in awareness and concentration be clearly differentiated. Students must have a clear conceptual and experiential knowledge of this difference. In Taming, concentration and awareness are two distinctly different forms of training that are gradually blended together. It is not until near the end of the book that I discuss what mindfulness is.

Because of the current popularity of mindfulness, one may choose to describe one's program or research in terms of mindfulness as long as the training clearly separates concentration and awareness. My publisher allowed me to keep my title Taming the Drunken Monkey as long as mindfulness could be included in their subtitle: The Path to Mindfulness, Meditation, and Increased Concentration.

\section{Breathwork}

In a one-time 4-hr breathwork workshop, students can learn the following: conceptually and experientially - the nature of a complete breath and health implications, the difference between chest breathing and diaphragm breathing, use of controlled breathing for relaxation or energy, and the use of controlled breathing as a self-control technique to reduce stress or unwanted emotions, such as anxiety or anger. All of this is done with strong components of concentration and awareness. The power of this training is that after just one workshop, the students have tools they can apply right away, often in significant ways.

In Taming, breathwork is a third major theme in addition to concentration and awareness. This includes the topics of the previous paragraph plus basic pranayama, the yogic science of breath.

I find that it is very effective to start with breathwork before beginning training in mindfulness/meditation. There are many diverse reasons for this, such as: (1) Students discover that they can learn powerful practical skills via breathwork. This is very motivating to continue additional training whose benefits will probably not come so soon. Contrast breathwork with training in concentration, where perceived improvement is often considered too slow for many North Americans. (2) Using controlled breathing to relax, before and during mental training, facilitates the cultivation of both concentration and awareness. (3) In Taming, vipassana, hatha yoga, and other approaches, the breath is an object of attention in early and, perhaps, later cultivation of concentration and awareness. By doing breathwork first, the student is already very familiar with breathing before beginning mental training. Thus, the student can focus more on the process of concentration and/or awareness with less distraction caused by learning about breathing and its lessons. This also makes it easier for the students to develop a clear experiential sense of exactly what concentration and awareness are. 
Although I like to begin concentration and awareness training using the breath as object, I soon move into also using a variety of other objects and activities. Ultimately, the students learn that almost every situation is an opportunity to further cultivate concentration and/or awareness. Of course, some situations are easier or more important than others.

In Taming, awareness of breathing is one component of learning to be more aware of the body. Other such practices are related to body scans, muscle relaxation, walking meditation, and pain.

\section{Attitude}

By attitude, I mean the mental set in which one approaches mental training in specific and living in general. Attitude includes moods, associations, expectations, and intentions. Attitude is one of the major components of meditation practice, a component that is always important, but has not received adequate attention (cf. Mikulas 2014a). The same is true of mindfulness development.

Attitude is thus the fourth major theme in Taming. The attitude components, which are gradually introduced, are in order: act with intention, have fun, make friends with yourself, be in the here-and-now, and not-doing. These attitude components strongly impact most mental training, including the development of concentration and awareness.

The instructions and examples of each attitude component vary with the students' level of development in mental training. A strength and beauty of these attitude components is their rich depth that is only gradually revealed through experience. At first, being in the here-and-now is a reminder to be more fully present in one's immediate experiences. Later, one finds there is only the here-andnow. At first, not doing is advice to become less goaloriented and switch from doing to being. Later, it is found that not-doing is a profound high teaching in many traditions. In mindfulness-based Zen meditation, one does not meditate to become enlightened. Rather, the meditation itself is an expression of enlightenment.

It is very important to realize that although attitude affects the development of mindfulness, no attitude components are part of the behavior of concentration or awareness. Confounding attitude and mindfulness can lead to theoretical and practical problems. For example, currently in North America, for historical and political reasons, some groups define mindfulness as including acceptance, but acceptance is independent and separate from concentration and awareness; acceptance can be something one is aware of. Also, the Buddha never included acceptance as part of mindfulness. Telling students that mindfulness requires acceptance will impair the development of awareness. For example, it will create a cognitive bias against being aware of non-acceptance, which could have clinical implications.

Currently, the two major confusions in the Western mindfulness literature are the confounding of concentration and awareness and confounding of these with attitude (Mikulas 2011). Some theorists propose that they are developing a Western psychological mindfulness, which is not the same as Buddhist mindfulness. This new mindfulness is some, as yet unspecified, combination of awareness, concentration, and acceptance. However, the point in this paper is that however one chooses to define mindfulness, in terms of development, it is very important to conceptually and experientially keep attitude separate from concentration and awareness.

\section{Questionnaire}

In cultivating mindfulness, it is helpful to use a mindfulness questionnaire before and after training. This allows the students to see their progress and identify areas that need more attention. Taming includes our awareness questionnaire that readers are encouraged to fill out right away and periodically retake as they progress through the program.

In cases of research or accountability, one can compute a general overall score for completed questionnaires and calculate changes in this score; but for the students, I recommend not coming up with a single score. Rather, in Taming and elsewhere, I suggest the students just compare changes in responses to each item and what can be learned.

There also are some subtle, but important, issues in trying to measure awareness. For example, let us say, I have some questionnaire items that relate to the students' awareness of images and dialogue in their minds. The unaware students may have little awareness of this monkey business and, hence, have low scores. Then, as they become more aware, the scores go up. Later, through concentration, they quiet some of the mental activities and the scores go back down. Combining such scores into a general measure of mindfulness has validity problems. However, there is no problem for the students to reflect on why their answers to specific items changed or not.

In our questionnaire, people answer most items on a 1 to 5 scale, but two other responses are possible: not applicable (NA) and the statement is unclear (X). Perhaps unique to our questionnaire, I have found the " $\mathrm{X}$ " response interestingly useful. Consider the item "I notice a difference between sound and the hearing of sounds." For many people, this item makes no sense, and they marked it X. Later, in their awareness training, they learn to experientially differentiate sound from the hearing. When they retook the questionnaire, they now understand the item that previously was not understandable. For many, this is a strong demonstration of their learning, which delights and/or empowers them. 


\section{Flexibility and Play}

In addition to the four themes discussed (concentration, awareness, breathwork, and attitude), there are two other themes that are traced through the different levels of Taming. These are mental flexibility and mental play.

Cultivating mental flexibility has two components, increasing flexibility and decreasing attachments. Increasing flexibility involves practices related to being wrong, questioning assumptions, lateral thinking, and creativity. Decreasing attachments focuses on the results of the clinging behavior of the mind. The mind has a tendency to grasp for and cling to certain contents of the mind, assumptions about self and reality, and personal frames of references. One can have attachments to perceptions, rituals, expectancies, opinions, images of the self, and models of reality.

Attachments distort perception and memory, impair thinking, and elicit undesired emotions. In Buddhism, the grasping and clinging that lead to attachments is understood to be the cause of most psychologically based suffering. Reducing attachments can significantly increase the clarity and breadth of awareness, and developing attachment-related awareness of various cues, such as body sensations, thoughts, and intentions, is an important part of reducing attachments.

The last theme is mental play, a series of optional activities to encourage playfully using one's mind. Included here are puzzles, paradoxes, games, philosophical speculations, and introspective queries.

\section{Not Religion}

In the beginning of Taming and throughout the book, I emphasize that the practices are not specifically religious or spiritual in many uses of these words. None of the practices is described within a religious context. One can profit from developing concentration and awareness whether one is religious or not. The program of Taming is not based on beliefs. One simply does the practices and sees for oneself.

This is the final example of the main point of this paper: When developing mindfulness, keep all the various related components experientially clean and separate. For example, do not let religious beliefs impair motivation or awareness, or distract from the basic practices. On the other hand, increasing mindfulness can improve anything that one does, so it must also facilitate religious and spiritual practices. Increasing awareness is the central Buddhist practice, and increased concentration can improve Christian prayer and Hindu devotion; it can create the space for the Christian Holy Spirit and Buddhist insight. Thus, in Taming, there are some optional sections showing how the basic practices relate to personal/ spiritual growth. Included are sections on meditation, awakening, transpersonal domains, and opening the heart. Opening the heart is a powerful way to reduce attachments and increase the breadth of awareness.

When we have a clear practical understanding of the distinctively different components related to the development of mindfulness, we can apply this powerful knowledge to any domain, including personal/spiritual growth, therapy, music, art, and sports. With mindfulness, you can improve how you do almost anything.

\section{References}

Cavanagh, K., Strauss, C., Forder, L., \& Jones, F. (2014). Can mindfulness and acceptance be learnt by self-help? A systematic review and meta-analysis of mindfulness and acceptance-based self-help interventions. Clinical Psychology Review, 34, 118-129.

Dunn, B. R., Hartigan, J. A., \& Mikulas, W. L. (1991). Concentration and mindfulness meditation: unique forms of consciousness? Applied Psychophysiology and Biofeedback, 24, 147-165.

Mikulas, W. L. (1981). Buddhism and behavior modification. Psychological Record, 31, 331-342.

Mikulas, W. L. (1990). Mindfulness, self-control, and personal growth. In M. G. T. Kwee (Ed.), Psychotherapy, meditation, and health. London: East-west.

Mikulas, W. L. (2000). Behaviors of the mind, meditation, and health. In K. T. Kaku (Ed.), Meditation as health promotion. Delft: Eburon.

Mikulas, W. L. (2002). The integrative helper: convergence of Eastern and Western traditions. Pacific Grove: Wadsworth.

Mikulas, W. L. (2011). Mindfulness: significant common confusions. Mindfulness, 2, 1-7.

Mikulas, W. L. (2014a). Meditation attitude. In N. N. Singh (Ed.), The psychology of meditation (pp. 29-43). New York: Nova.

Mikulas, W. L. (2014b). Taming the drunken monkey: the path to mindfulness, meditation, and increased concentration. Woodbury: Llewellyn. 